\title{
La réforme du système éducatif angolais en 2016
}

Un aperçu du contexte

\section{Pierre Homerin}

\section{CpenEdition}

\section{Journals}

Édition électronique

URL : https://journals.openedition.org/ries/5582

DOI : $10.4000 /$ ries.5582

ISSN : 2261-4265

Éditeur

France Education international

Édition imprimée

Date de publication : 1 décembre 2016

Pagination : 24-30

ISBN : 978-2-85420-612-8

ISSN : $1254-4590$

Référence électronique

Pierre Homerin, "La réforme du système éducatif angolais en 2016 », Revue internationale d'éducation de Sèvres [En ligne], 73 | décembre 2016, mis en ligne le 01 décembre 2018, consulté le 01 juillet 2021 URL : http://journals.openedition.org/ries/5582 ; DOI : https://doi.org/10.4000/ries.5582

Ce document a été généré automatiquement le 1 juillet 2021.

(c) Tous droits réservés 


\section{La réforme du système éducatif angolais en 2016}

Un aperçu du contexte

Pierre Homerin

1 L'Angola est une île. Avec près de $2700 \mathrm{~km}$ de frontières le séparant des pays francophones du nord, le Congo $(200 \mathrm{~km})$ et la République démocratique du Congo $(2511 \mathrm{~km})$, et près de $2500 \mathrm{~km}$ des pays anglophones de l'est (Zambie, $1110 \mathrm{~km})$ et du sud (Namibie, $1376 \mathrm{~km}$ ), l'Angola revendique son originalité lusophone, accentuée par l'indépendance obtenue en 1975, par le soutien des pays d'Europe de l'Est et de Cuba lors de la guerre civile jusqu'à la paix de 2002, et par les liens économiques tissés aujourd'hui avec le Brésil et la Chine. Les systèmes de pensée s'y côtoient et s'y opposent, à partir d'une forte tradition portugaise assumée avec méfiance, alimentés par une formation des cadres et par des réseaux universitaires cosmopolites, pour développer un système éducatif qui se veut original et national, associant les meilleures pratiques internationales.

2 En 2014, le premier recensement depuis l'indépendance de 1974 fait état d'une population de plus de 25,8 millions d'habitants ${ }^{1}$, à majorité urbaine $(62,3 \%)$ et féminine (52\%). En attendant une nouvelle réforme territoriale en cours, le pays est divisé en dix-huit provinces, dont la densité démographique moyenne est de 20,7 habitants au $\mathrm{km}^{2}$. L'âge moyen de la population s'élève à 20,6 ans, dont $47,3 \%$ de jeunes de moins de 14 ans et $2,4 \%$ de plus de 65 ans. Si, du fait du flot de réfugiés issu de trente ans de guerre civile, plus d'un quart de la population se concentre dans la province de Luanda (6,5 millions d'habitants, 368 habitants au $\left.\mathrm{km}^{2}\right)$, aujourd'hui, les provinces centrales de Huambo, Bié et Moxico possèdent une population dont plus de la moitié a moins de 14 ans. En Angola, plus de $65 \%$ de la population a moins de 24 ans !

Dans les dix années qui ont suivi l'accord de paix de 2002, l'Angola a connu une période de forte croissance économique qui, malgré une corruption prédatrice ${ }^{2}$ et des enrichissements personnels colossaux, a néanmoins permis de ramener le taux de population vivant avec moins de deux dollars par jour à $54 \%$, pour une espérance de vie de 51,9 ans en 2013 (contre $92 \%$, pour 45,2 ans en 2000). 
Cette croissance est essentiellement due à la production pétrolière, qui représente $43 \%$ du PIB, $70 \%$ des recettes fiscales et $96 \%$ des exportations (2013). Mais cette dépendance le rend également très fragile et le pays a vu sa progression considérablement freinée lors de la crise mondiale de 2008 et surtout depuis 2014, avec la baisse de plus de $60 \%$ des cours du baril de pétrole. Le budget de l'état a été ramené de 72 à 54 milliards de dollars en 2015, conduisant à l'arrêt de nombreux travaux et investissements. Toutefois, la défense et la sécurité du pays restent prioritaires avec $15 \%$ des dépenses totales, toujours supérieures à celles consacrées à la santé et à l'éducation (13\%).

\section{Statistiques en éducation}

5 Lors des années fastes, des investissements structurels considérables ont été faits dans tous les secteurs, et notamment dans ceux de l'éducation et de l'enseignement supérieur, appuyés également par l'Union européenne, l'Unesco et la Banque Mondiale.

6 Le pays est passé de 17852 salles de classe en 2001 (14 117 dans le primaire, 2398 dans le secondaire inférieur et 1337 dans le secondaire supérieur), à plus de 82000 en 2015 (64 600 dans le primaire, 12282 dans le secondaire inférieur et 5148 dans le secondaire supérieur), ce qui a permis de porter le nombre d'élèves à plus de huit millions ${ }^{3}$ à la rentrée 2015, pour un total de 8,309 millions d'apprenants dans l'ensemble des soussystèmes éducatifs.

7 Néanmoins, le déficit reste considérable : $13,4 \%$ des jeunes de moins de 18 ans n'ont jamais fréquenté l'école et seulement $13 \%$ des jeunes de 18 à 24 ans ont pu terminer des études secondaires. Si le taux de scolarisation dans le primaire a fortement progressé, il n'est encore que de $76 \%$ et, surtout, seuls $30 \%$ le terminent. Enfin, pour l'ensemble du pays, 2,5\% des personnes de plus de 24 ans déclarent posséder une formation supérieure (5,4 \% à Luanda), sans qu'il soit possible d'en préciser la qualité ${ }^{4}$.

\section{Les lois de base du système éducatif}

\section{Avant 2016}

8 Après un premier système d'urgence en 1977, destiné à rompre avec l'héritage colonial portugais, en adoptant les principes de démocratisation et de gratuité de l'enseignement qui permirent de scolariser 1800000 élèves en 1980, dans des conditions souvent précaires, une Loi de base pour le système éducatif a été adoptée le 31 décembre 2001, dans une optique de transition d'une économie socialiste vers une économie de marché, et d'adaptation de l'individu aux exigences de la vie politique, économique et sociale du pays.

9 Cette loi réaffirme les principes fondamentaux de la laïcité, de l'accès démocratique, de la gratuité et de la scolarité obligatoire de l'enseignement primaire et distingue six sous-systèmes: l'éducation préscolaire, l'enseignement général, l'enseignement technique-professionnel, la formation des enseignants, la formation des adultes et l'enseignement supérieur.

Elle met en œuvre une réforme en cinq étapes: une nouvelle organisation des institutions, de nouveaux programmes, expérimentaux, la généralisation de ceux-ci, l'évaluation du système et enfin, les objectifs fixés, notamment qualitatifs, n'ayant pas 
été atteints l'élaboration d'une nouvelle Loi de base, approuvée définitivement le 11 août 2016.

\section{La nouvelle loi de base de 2016}

11 Cette nouvelle loi confirme la philosophie et de nombreuses dispositions de la précédente, avec pour objectif de construire un système éducatif cohérent, en coordonnant les six sous-systèmes d'enseignement.

12 L'organisation générale des institutions de l'enseignement primaire, secondaire et supérieur est précisée, ainsi que l'articulation entre les sous-systèmes, avec l'introduction de limites d'âge pour chacun. Les principaux changements portent sur l'extension de la scolarité obligatoire et de la gratuité jusqu'au premier cycle de l'enseignement secondaire ( $9^{\mathrm{e}}$ année, à 14 ans), et la réglementation des frais d'inscription pour le second cycle de l'enseignement secondaire et l'enseignement supérieur.

13 Outre le portugais, qui continue d'être langue d'enseignement obligatoire, parlée par $85 \%$ de la population urbaine, le rôle des autres langues est renforcé, tant dans l'enseignement (les langues nationales, très présentes dans les zones rurales $(50 \%)$, le langage des sourds-muets) qu'en ce qui concerne le développement de politiques publiques en faveur des principales langues dites de « communication internationale », l'anglais et le français.

14 Une nouvelle modalité apparaît, l'enseignement semi-présentiel, afin de faire place aux nouvelles technologies.

15 Enfin, la loi introduit deux années de «travail obligatoire dans le domaine de formation " à la suite de la quatrième année à visée professionnelle de l'enseignement secondaire, pour les étudiants n'ayant pas accompli leur service militaire, ou à la fin de chaque niveau de l'enseignement supérieur, avant de pouvoir accéder au niveau suivant. Cette mesure, destinée à favoriser l'employabilité des étudiants mais aussi à désengorger les formations trop généralistes - et souvent privées - des universités après l'explosion démographique des années 2010-2015, risque néanmoins de provoquer de nouvelles frustrations dans une population estudiantine qui s'est précipitée dans le rêve d'emplois abondants et surpayés dans le secteur pétrolier ou dans la fonction publique.

\section{L'organisation du système}

\section{L'éducation préscolaire}

Destiné aux enfants de trois à six ans, le «jardin d'enfants" se termine par une dernière année en théorie obligatoire (classe d'initiation). Dans les faits, très peu d'établissements publics ont la capacité d'offrir cette classe souvent payante.

\section{L'enseignement général}

Il comprend l'enseignement primaire, d'une durée de six ans, et l'enseignement secondaire, lui-même subdivisé en deux cycles de trois années. 

années à la suite du premier cycle du secondaire ; or cet objectif est battu en brèche car 
les étudiants luttent pour obtenir le diplôme le plus élevé possible garantissant une rémunération et sont peu nombreux à se destiner à la carrière de professeur? pour adultes repose davantage sur une conception de rattrapage scolaire que sur celle d'une véritable éducation destinée aux adultes en activité. En effet, le même système et les mêmes programmes que ceux de l'enseignement général y sont reproduits, mais de façon concentrée, généralement en cours du soir : primaire découpé en alphabétisation et post-alphabétisation, à partir de méthodes cubaine et brésilienne, deux cycles de l'enseignement secondaire de trois années chacun.

Encore embryonnaire, la direction nationale pour la formation des adultes du ministère de l'éducation se voit confrontée à l'immense défi d'alphabétiser les populations qui ont souffert de la guerre civile et les jeunes qui n'ont pas eu accès au système scolaire. Grâce à ses efforts et avec l'aide de la société civile (ONG, associations communautaires, institutions religieuses, militaires, etc.), le taux d'alphabétisation du pays est aujourd'hui de $73 \%$ (contre moins de $50 \%$ en 2000$)^{8}$. Sur les huit millions d'apprenants de 2015, 781000 adultes ont bénéficié d'enseignements. ${ }^{9}$ 

système, avec une formation davantage liée aux besoins effectifs des adultes et qui
prenne en compte leurs contraintes pour des conditions d'enseignement spécifiques et
mieux adaptées.

\section{L'enseignement supérieur} Neto (UAN) de Luanda, qui possédait quelques extensions en province. Une réforme structurelle, initiée en 2007 et consolidée ces trois dernières années, a profondément modifié l'enseignement supérieur angolais afin de répondre à une forte pression sociale et de donner au pays les ressources humaines nécessaires à son fort développement économique.

les facultés des universités, aboutissant aux diplômes traditionnels de «bacharelato » (trois à quatre ans) ou « licenciatura » (quatre à six ans), puis « mestrado » en deux ans. L'enseignement polytechnique, plus novateur, articule bacharelato et licenciatura dans les écoles ou instituts supérieurs polytechniques, pour une formation en deux étapes (3+2 années) permettant l'aller-retour dans l'emploi.

Néanmoins, avec la croissance exponentielle des effectifs et des établissements, le déficit d'enseignants qualifiés s'est accentué, et l'État angolais se voit confronté au défaut de qualité des enseignements et des institutions ainsi qu'à la faible employabilité des diplômés du système. Ceci a conduit à mettre en place un ambitieux Plan national de formation des cadres 2013-2020, visant particulièrement le ministère de l'enseignement supérieur avec, pour priorités, le renforcement du panorama des formations supérieures, en particulier technologiques, nécessaires au développement économique du pays, la promotion d'un corps d'enseignants universitaires démultiplicateurs de compétences et l'amélioration de la qualité des formations et des établissements du système d'enseignement supérieur. Malgré cet effort, les emplois promis seront-ils au rendez-vous?

\section{Les circuits de coordination, de régulation, de contrôle et d'évaluation}

Ces réformes sont accompagnées de modifications de structures : l'unique secrétariat d'État à l'enseignement supérieur rattaché au ministère de l'éducation de 2001 est devenu ministère à part entière, lui-même scindé par la suite en ministère de l'enseignement supérieur (MES) et ministère de la science et de la technologie.

Au sein du MES, la création de deux nouveaux secrétariats d'État, l'un à la supervision et l'autre à l'innovation, confirme la volonté de consolider les réformes et de soutenir 
les initiatives visant à l'amélioration de la qualité de l'enseignement supérieur sur l'ensemble du pays.

41 La qualité du système éducatif et son rôle comme facteur de développement du pays sont au cœur des ambitions de la nouvelle loi. Afin de suppléer aux besoins de la société et aux revendications étudiantes pour une meilleure formation garantissant l'emploi, le gouvernement reconnaît la place importante laissé à l'initiative privée, mais, par ailleurs, impose un système unique de façon beaucoup plus précise, en couvrant la totalité du champ éducatif (enseignement spécial, enseignement à distance, à temps partiel...), multiplie les garde-fous et les instruments de contrôle pour garantir l'existence d'un système proprement angolais et limiter les dérives, souvent apportées par l'importation ex abrupto de modèles étrangers.

Cette nouvelle loi, et notamment la séparation en groupes d'âge, ambitionne de construire un véritable système capable de scolariser la totalité de la population du pays. L'Angola veut ainsi se donner les moyens d'atteindre l'objectif de $90 \%$ de jeunes alphabètes en 2020.

\section{BIBLIOGRAPHIE}

DOS SANTOS BENEDITO N. D. (2007) : Centralização de sistemas educativos e autonomia dos actores organizacionais, Universidade do Minho-Portugal.

CONSEIL DE DIRECTION DU MINISTÈRE DE L'EDUCATION (2011) : Balanço da Implementação da Reforma Educativa, mai, Ministère de l'éducation.

COMMISSÃO DE ACOMPAHAMENTO DAS ACÇÕES DA REFORMA EDUCATIVA (2010) : Relatório da fase de experimentação da Reforma Educativa, septembre, Ministère de l'éducation.

NASCIMENTO CHIMUCO S.-M. (2015) : A formação incial de professores em Angola no contexto da reforma educativa : desafios e necessidades, Universidade do Minho-Portugal.

\section{NOTES DE FIN}

1. Pour 5,6 millions au recensement précédent de 1970.

2. L'Angola est classé 165e sur 174 pays à l'indice de Transparency International (2014).

3. Statistiques MED (ministère de l'éducation), 2016, ANGOP.

4. INE : Recensement 2014.

5. $24 \%$ des enfants de 5 à 11 ans sont encore exclus du système scolaire (INE 2014).

6. Seuls $13 \%$ des jeunes de plus de 18 ans ont terminé l'enseignement secondaire (INE 2014).

7. Une étude menée à l'EFP de Cabinda en 2014 indique que 21,6 \% des diplômés seulement sont devenus enseignants. D'origine très modeste, ils voient dans cette formation le seul moyen de quitter leur état de pauvreté et sont très assidus mais déchantent rapidement. À l'EFP de Lubango, deuxième ville du pays, sur 1047 élèves-professeurs inscrits, 749 ont terminé leur première année, 234 ont redoublé et 63 ont quitté l'école.

8. Discours sur l'état de la nation (2014), chiffres PNUD. 
9. Nombre d'apprenants : préscolaire : 727000 ; primaire : 5,07 millions ; 1er cycle : 1,08 million ; 2nd cycle : 621700 .

10. Adão do Nascimento, ministre de l'enseignement supérieur, avril 2016.

\section{INDEX}

Mots-clés : politique éducative, réforme de l'enseignement, système éducatif Index géographique : Angola

Palabras claves : política educacional, reforma de la educación, sistema educativo Keywords : educational policy, educational reform, educational system

\section{AUTEUR}

\section{PIERRE HOMERIN}

Pierre Homerin est chargé de programmes au Centre international d'études pédagogiques (CIEP, France). Diplômé ès lettres de l'Université de Paris-X Nanterre, une grande partie de sa carrière s'est déroulée dans les pays lusophones : enseignant au Brésil, attaché de coopération pour le français au Portugal, chef de projet au Mozambique, attaché de coopération éducative en Angola. Conseiller en formation continue en France et directeur régional du SENAI au Brésil, il a développé une expertise en formation professionnelle et mène actuellement le projet de mise en place du Système national de certifications au Mozambique pour la Banque mondiale. Courriel : homerin@ciep.fr 